\title{
Systematic study of the effect of HSE functional internal parameters on the electronic structure and band gap of a representative set of metal oxides
}

\author{
Francesc Viñes, ${ }^{1}$ Oriol Lamiel-García, ${ }^{1}$ Kyoung Chul Ko, ${ }^{* 1,2}$ Jin Yong Lee ${ }^{2}$ and Francesc Illas* ${ }^{1}$
}

\author{
${ }^{1}$ F. Viñes, O. Lamiel-García, F. Illas \\ Departament de Ciència de Materials i Química Física \& Institut de Química Teòrica i Computacional (IQTCUB), \\ Universitat de Barcelona, c/ Martí i Franquès 1, Barcelona, Spain, 08028 \\ E-mail: francesc.illas@ub.edu \\ ${ }^{2}$ K. C. Ko, J. Y. Lee \\ Department of Chemistry, Sungkyunkwan University, Suwon, Korea, 16419 \\ E-mail:kcko1982@gmail.com
}

\begin{abstract}
The effect of the amount of Hartree-Fock mixing parameter $(\alpha)$ and of the screening parameter $(w)$ defining the range separated HSE type hybrid functional is systematically studied for a series of seven metal oxides: $\mathrm{TiO}_{2}, \mathrm{ZrO}_{2}, \mathrm{CuO}_{2}, \mathrm{ZnO}, \mathrm{MgO}, \mathrm{SnO}_{2}$, and $\mathrm{SrTiO}_{3}$. Firstly, reliable band gap values were determined by comparing the optimal $\alpha$ reproducing the experiment with the inverse of the experimental dielectric constant. Then, the effect of the $w$ in the HSE functional on the calculated band gap was explored in detail. Results evidence the existence of a virtually infinite number of combinations of the two parameters which are able to reproduce the experimental band gap, without a unique pair able to describe the full studied set of materials. Nevertheless, the results point out the possibility of describing the electronic structure of these materials through a functional including a screened HF exchange and an appropriate correlation contribution.
\end{abstract}

\section{Introduction}

Metal oxides constitute the most abundant materials on Earth crust $^{1}$ and are attracting widespread interest in various scientific fields, from electronics to heterogeneous catalysis, passing through the fascinating, yet not fully understood, phenomenon of high critical temperature superconductivity discovered back in 1986 by Bednorz and Müller. ${ }^{2}$ Applications involving metal oxides cover the broad ranges on thin-film transistors, solar cells, diodes, memories, and photocatalysts. ${ }^{3-5}$ The properties of metal oxides are highly dependent on their electronic structure; for instance, the band gap plays a key role in optoelectronic properties and is also crucial in applications such as photocatalysis. In this context, band gap engineering becomes one of the important issues for the application of metal oxides in light energy harvesting for energy/environment sciences and technologies. ${ }^{6}$

The band gap of these materials is often described in the framework of band structure theory and most often numerical predictions rely on different implementations of conventional density functional theory (DFT). However, the exchange-correlation potentials derived from the commonly used local density approximation (LDA) or generalized gradient approximation (GGA) based functionals tend to deliver severely underestimated band gaps for metal oxides to the point that calculations may incorrectly predict a metallic behavior for experimentally confirmed semiconductors. ${ }^{7,8}$ This wrong behavior of LDA and GGA based methods is usually referred to as the band gap problem. ${ }^{9}$ 
Basically, the band gap problem arises from the usage of grossly approximated potentials under the Kohn-Sham implementation, and so, suffer from the self-interaction error (SIE) ${ }^{10-11}$ which is intrinsic to the way the Coulomb contribution is accounted for. Thus, the LDA and GGA calculated results are intrinsically affected by the SIE. In order to reduce SIE, which is exactly cancelled in Hartree-Fock (HF) method, mixing of LDA or GGA exchange potentials with HF non-local exact exchange term has been proposed. This choice leads to the so-called hybrid functionals, first proposed by Becke. ${ }^{12}$ In hybrid functionals normally a certain percentage of semilocal exchange, ranging from 0.1 to 0.5 (10 to $50 \%$ ), is replaced by exact HF exchange, while the pure semilocal correlation potential is maintained. Some of the most widely used hybrid functionals, such as B3LYP ${ }^{12}$ and PBEO, ${ }^{13,14}$ containing 20 and $25 \%$ of $\mathrm{HF}$ exchange, respectively, have been found to be successful in accurately describing the geometrical and thermodynamic properties for a wide range of molecular systems. ${ }^{11}$ However, in solids, the non-local nature of the Coulomb operator in the HF exchange of hybrid functionals leads to technical difficulties regarding slow convergence ${ }^{15}$ as well as highly expensive computational cost, especially when using a plane waves basis set although with excellent performance. ${ }^{16}$

Nevertheless, using a standard gaussian type orbitals (GTO) basis set, hybrid functionals have been successfully used to predict band gaps of transition metal oxides ${ }^{17-19}$ and of more complicated systems such as antiferromagnetic $\mathrm{NiO} .{ }^{20}$ Hybrid functionals using a plane wave basis sets have also been successfully applied to magnetic systems such as $\mathrm{MnO}^{21}$ and to stoichiometric and reduced $\mathrm{CeO}_{2}{ }^{22}$ However, the latter study uses a range separated exchange-correlation functional ${ }^{23}$ which has been introduced to overcome the convergence drawbacks of hybrid functional above mentioned. In addition to the amount of Fock exchange, the range separated functionals introduce a second parameter aimed to define the short-range or long-range domains as described in more detail in the next section. The presence of this second parameter in the exchange-correlation potential results in a rather accurate description of the structural and thermochemical properties of both molecules and solids, including energy band gaps of solids ${ }^{23-30}$ but at the same time introducing an additional degree of empiricism which may, in addition, introduce biases or artifacts in the calculated results. Previous studies focused on the average error introduced from different choices of these parameters. ${ }^{31}$ Here, we provide an alternative point of view by systematically exploring the combinations of the parameters reproducing experimental properties. In particular, we will show that for certain observables, such as the band gap, there is a multitude of combinations of both parameters matching the experimental result. The consequences of this behavior are also described in the forthcoming sections.

\section{Brief description of the HSE range separated hybrid functional}

The HSE functional grounds on Perdew-BurkeErnzerhof $(P B E)^{32}$ flavor of the GGA family of functionals, where the exchange-correlation energy is separated into exchange and correlation parts:

$E_{x c}^{P B E}=E_{x}^{P B E}+E_{c}^{P B E}$

Mixing the PBE exchange potential with a given fraction of the non-local exact HF exchange, generates the PBEO hybrid functional. ${ }^{13}$ Here one has

$E_{x c}^{P B E 0}=\alpha E_{x}^{H F}+(1-\alpha) E_{x}^{P B E}+E_{c}^{P B E}$

where $\alpha$ indicates the HF mixing parameter. The PBEO functional uses $\alpha$ as $1 / 4$, a non-empirical coefficient justified from perturbation theory. ${ }^{13,33}$ Nevertheless, this choice is not appropriate to describe $\mathrm{NiO}^{20}$ or the different polymorphs of stoichiometric and reduced titania $\left(\mathrm{TiO}_{2}\right)^{34}$ where higher and smaller amounts of Fock exchange are required to 
reproduce experimental values of electronic structure dependent properties, respectively.

To avoid the cumbersome convergence of hybrid functionals in some periodic approaches, mainly due to the problematic long-range HF exchange, the HSE functional introduces a screened Coulomb potential, which only affects the exchange part of the exchange correlation potential. $^{23,27}$ Thus, the exchange part of the electron-electron Coulomb interaction is arbitrarily decomposed into short range (SR) and long range (LR) contributions, and screened using the error function $\operatorname{erf}(r)$ as in Eq. (3)

$\frac{1}{r}=S R+L R=\frac{e r f c(w r)}{r}+\frac{e r f(w r)}{r}$

where $\operatorname{erfc}(w r)=1$-erf $(w r)$ and $w$ is the screen parameter. $^{35}$ Henceforth, the HSE screened hybrid functional takes the form as in Eq. (4) ${ }^{36}$

$E_{x C}^{H S E}=\alpha E_{x}^{H F, S R}(w)+(1-\alpha) E_{x}^{P B E, S R}(w)+$ $E_{x}^{P B E, L R}(w)+E_{c}^{P B E}$

Thus, the HSE functional contains two parameters: the HF mixing parameter $\alpha$ and the screen parameter $w$. Note also that HF exchange mixing involves the $S R$ part of the exchange potential only. In the limiting case of $w=0$, the HSE functional becomes PBEO, whereas for $w \rightarrow \infty$ it becomes PBE. For the standard HSE functional, usually referred to also as HSE06, $\alpha$ and $w$ values were empirically defined as 0.25 and $0.21 \AA^{-1}\left(0.11\right.$ Bohr $\left.^{-1}\right)$, respectively, chosen by considering both accuracy of results and computational efficiency, as suggested by Scuseria et al. ${ }^{24,27}$

Coming back to the band gap problem, it is known that while LDA and GGA significantly underestimate the band gap of oxides and related materials, the increasing of $\mathrm{HF}$ exchange ( $\alpha$ in Eq. 4 ) increases the calculated band gap. ${ }^{37}$ Previous studies suggested that the optimal $\alpha$ for $\mathrm{NiO}$ was $\sim 0.35,{ }^{20}$ whereas a smaller value of 0.125 is necessary to reproduce the band gap of anatase and rutile polymorphs of $\mathrm{TiO}_{2}{ }^{34}$ Recently, a modification of the two parameters was also tried to adjust the calculated band gap of $\mathrm{TiO}_{2}$ materials. ${ }^{38}$ In a similar way, $\mathrm{Ha}$ et al. used $\alpha=0.25$ and $w=\sim 0.378 \AA^{-1}\left(0.200 \mathrm{Bohr}^{-1}\right)$ to reproduce the band gap of $\mathrm{TiO}_{2}$ anatase, whereas Janotti et al. used $\alpha=0.20$ and $w=$ $0.200 \AA^{-1}\left(0.106\right.$ Bohr $\left.^{-1}\right)$ for rutile $\mathrm{TiO}_{2}{ }^{39}$ In this context, it is clear that different choices of the $\alpha$ and $w$ parameters are possible, and this may be a convenient way to accurately predict the band gap of metal oxides. The role of the two parameters in the HSE functional was studied in detail by Moussa et al. ${ }^{31}$ These authors studied the effect of the two parameters in a total of 33 solids, including metals, semiconductors, and insulators but only one metal oxide. The main focus of this work was in the error trends regarding several physical properties arising from different choices of the amount of Fock exchange and screening parameters. Afterwards, Koller et al. kept $w$ fixed at $0.312 \AA$ ${ }^{1}$ while optimized $\alpha$ for several solids including 5 metal oxides. ${ }^{40}$ More recently, a non-empirical way to define the optimal parameters in range separated hybrid functionals was carried out by Galli et al., ${ }^{41}$ yet the full space of $w$ and $\alpha$ combinations was not fully explored.

Despite the many studies above mentioned addressing the two parameters in HSE, studies on practical combinations for different values of $\alpha$ and $w$ parameters targeting the experimental band gaps are rare, with most of the focus aimed at showing the least mean absolute error for each set of parameters. Moreover, in many studies some parameters were fixed to a certain value sparsely. Furthermore, a limited number of metal oxides was investigated, whereas the electronic structure of these materials is known to be specially challenging. ${ }^{9}$ For this reason we here make as a further step paying attention to disclose the effect of choice of two parameters by systematically analyzing the overall $\alpha$ and $w$ combinations reproducing reliable experimental band gaps for seven metal oxides, using scalar relativistic all electron density functional calculations. Our study would help to answer the question of how a set of optimal parameters is distributed, and whether a universal parameter exists among the 
investigated metal oxides. These results aid at understanding the effect of the HSE functional internal parameters on calculated band gaps of metal oxides. In addition, the rational procedure to gain these combinations can, by extension, be easily applied to other solid systems within HSE formalism.

\section{Material models and computational details}

In order to investigate the existence of an optimal combination of Hartree-Fock mixing and screening parameters, $\alpha$ and $w$, respectively, up to seven metal oxides systems were considered in this study. These are $\mathrm{TiO}_{2}$ (anatase), $\mathrm{SrTiO}_{3}$ (cubic), $\mathrm{Cu}_{2} \mathrm{O}$ (cubic), $\mathrm{SnO}_{2}$ (rutile), $\mathrm{ZrO}_{2}$ (monoclinic), $\mathrm{ZnO}$ (wurzite), and $\mathrm{MgO}$ (cubic). These metal oxides were selected from previous studies reporting their electronic structure description, the choice of $\alpha$ in hybrid functionals following arguments based on the dielectric constant $(\varepsilon \infty),{ }^{31,40-43}$ or their interest for the photocatalytic water splitting. ${ }^{44}$

To eliminate the structural effect on the calculated band gaps, we used the experimentally obtained structures taken from the inorganic crystal structure database (ICSD) ${ }^{45}$ through the Materials Project webpage, $^{46}$ see Table S1 in Supporting Information. It is notable that effect of $\alpha$ parameter is large on the electronic structure and, hence the band gap but much smaller in change of atomic structure, as reported for $\mathrm{TiO}_{2}$ cases. ${ }^{34}$ Single point calculations were carried out using $7 \times 7 \times 7$ Monkhorst-Pack mesh of special k-points except for $\mathrm{MgO}$ where a $17 \times 17 \times 17$ k-points Monkhorst-Pack mesh was used just to further check convergence of the electronic structure with respect to integration in the reciprocal space. All periodic density functional calculations were performed using the FHI-aims electronic structure code. ${ }^{47,48}$ Present calculations explicitly include all electrons and the electron density is described by means of numerical atom-centered orbitals (NAO). ${ }^{49}$ The light grid in combination with the
Tier-1 basis set was chosen for its accuracy similar to or higher than that obtained with 6311G* and just below of TZVP quality. The accuracy of the basis set can be inspected by just focusing on the total energy since, according to the variational theorem, the lower the total energy the more complete the basis set is and the total energy closer to the exact variational solution. For instance, for the $\mathrm{O}_{2}$ molecule in its ground triplet state at a distance of $1.156 \AA$, the light Tier-1 total energy for nonrelativistic at the unrestricted HF (UHF) level is $4072.582 \mathrm{eV}$, significantly lower than the corresponding value of $-4072.456 \mathrm{eV}$ obtained with the 6-311G*. This is also the case for the $\mathrm{TiO}_{2}$ molecule in its singlet ground state at a given geometry (with $\mathrm{Ti}-\mathrm{O}$ distance of $1.5977 \AA$ and O-Ti-O angle of $115.96^{\circ}$ ) for which HF nonrelativistic total energy with light Tier-1 is $27160.764 \mathrm{eV}$ and the corresponding nonrelativistic HF value for the $6-311 \mathrm{G} *$ basis set is $-27159.604 \mathrm{eV}$. For the $\mathrm{Ti}$ atom in the triplet state the light Tier-1 basis set leads to an UHF total energy of $-23085.533 \mathrm{eV}$ meanwhile with the $6-311 G^{*}$ basis set the total UHF energy is $23084.972 \mathrm{eV}$. With this, the $\mathrm{TiO}_{2}$ energy formation starting from $\mathrm{Ti}$ and $\mathrm{O}_{2}$ is $-2.649 \mathrm{eV}$ for the light Tier-1 basis set and $-2.176 \mathrm{eV}$ for the $6-311 \mathrm{G}^{*}, 0.473 \mathrm{eV}$ smaller than the value obtained from the light Tier-1 basis set. Note also that, except for these calculations for the $\mathrm{O}_{2}$ and $\mathrm{TiO}_{2}$ molecules aimed at providing information about the quality of the NOA basis sets used, all calculations described in the present work include scalar relativistic effects through the ZORA method, ${ }^{50}$ omitted in previous works or included through a pseudopotential. ${ }^{31}$

To search for a possible optimal combination of $\alpha$ and $w$ that can best reproduce the experimental band gap value of these oxides we systematically screened the two parameters. Thus, $w$ values of $0.0,0.2,0.4,0.6,0.8$, and 1.2 $\AA^{-1}$ were considered, and for each of them $\alpha$ was varied from 0 to 1 with 0.25 intervals. Moreover, additional calculations were carried out to refine some points corresponding to $w$ 
parameters of $0.6 \AA^{-1}$ or $0.9 \AA^{-1}$ to find the optimal $\alpha: w$ curve trend. This curve corresponds, for each oxide, to the combination of $\alpha$ and $w$ values which reproduce the experimental band gap. Here a caveat is necessary since one has to regard that the band gap is computed from the band structure obtained from the Kohn-Sham orbital energies in the reciprocal space rather than from using the theoretically more grounded many-body quasi particle based GW formalism. Band structure calculations are usually performed at DFT level when GW method is not affordable. Moreover, the success of hybrid and range separated hybrid functionals in predicting the band gap is precisely based on the assumption that this property can be extracted from the band structure.

\section{Results and discussion}

In agreement with our previous study on bulk $\mathrm{TiO}_{2}$ rutile and anatase structures, ${ }^{34}$ we found that $\alpha$ and the calculated band gap exhibit a linear relationship good enough to use linear inter/extrapolation to find, for a certain fixed value of $w$, the $\alpha$ value matching the experimental band gap (see Figure S1 in Supporting Information). In the following, we refer to this $\alpha$ value as optimal. On the other hand, for a fixed $\alpha$, the screen parameter $w$ and the calculated band gap are roughly in inverse proportion to each other, but without displaying a linear relationship, especially for cases of high $\alpha$ values where an exponential decay trend is observed. Thus, in this study, we decided to use the relationship between the $\alpha$ parameter and the calculated band gap to find the optimal combination of $\alpha$ and $w$ also with respect to the experimental band gap. In the following we discuss several relationships regarding the mixing parameter $\alpha$, the dielectric constant $(\varepsilon \infty)$, the band gap, and the optimal combinations of $\alpha$ and $w$.

\section{Defining the optimal mixing parameter without screening effect}

First of all, we discuss the results obtained by varying the $\alpha$ parameter while keeping $w=0$ and compare to the experimental band gaps as reported in Table 1 -with ranges given in parenthesis. It should be noted except for $\mathrm{Cu}_{2} \mathrm{O}$, the experimental value used to obtain the optimal $\alpha$ is the lowest value in the experimental range in Table 1.

\begin{tabular}{|c|c|c|c|c|c|}
\hline & $\Delta$ & $\alpha$ & $\varepsilon^{\infty}$ & $1 / \varepsilon^{\infty}$ & $w$ \\
\hline $\mathrm{Cu}_{2} \mathrm{O}$ & $\begin{array}{c}1.57^{[a]} \\
(1.70-2.40)^{51-54}\end{array}$ & 0.119 & $6.46^{55}$ & 0.155 & 1.21 \\
\hline $\mathrm{TiO}_{2}$ & $\begin{array}{c}3.20^{[b]} \\
(3.20-3.42)^{56,57}\end{array}$ & 0.134 & $5.62^{58}$ & 0.178 & 1.29 \\
\hline $\mathrm{SrTiO}_{3}$ & $\begin{array}{c}3.25 \\
(3.25-3.75)^{59}\end{array}$ & 0.154 & $5.18^{60}$ & 0.193 & 1.14 \\
\hline $\mathrm{ZrO}_{2}$ & $\begin{array}{c}5.30 \\
(5.30-7.09)^{61,62}\end{array}$ & 0.181 & $4.54^{63}$ & 0.220 & 0.94 \\
\hline $\mathrm{SnO}_{2}$ & $\begin{array}{c}3.30^{64} \\
(3.30-4.00)^{64}\end{array}$ & 0.207 & $4.00^{65}$ & 0.250 & 0.94 \\
\hline Zno & $\begin{array}{c}3.37^{66} \\
(3.37-3.44)^{66-68}\end{array}$ & 0.254 & $3.74^{69}$ & 0.267 & 0.88 \\
\hline MgO & $\begin{array}{c}7.67 \\
(7.67-7.83)^{70,71}\end{array}$ & 0.293 & $2.96^{71}$ & 0.338 & 0.7 \\
\hline
\end{tabular}

[a] Estimated value considering the energy correction around $0.13 \mathrm{eV}$ from the experimental band gap $1.7 \mathrm{eV}$.

[b] Consensus value.

For $\mathrm{Cu}_{2} \mathrm{O}$, we estimated the experimental band gap value with consideration of the energy correction for the forbidden transition as discussed in more detail below. The present calculations predict direct band gaps for $\mathrm{Cu}_{2} \mathrm{O}$, $\mathrm{SnO}_{2}, \mathrm{ZnO}$, and $\mathrm{MgO}$, and indirect band gaps for $\mathrm{TiO}_{2}, \mathrm{SrTiO}_{3}$, and $\mathrm{ZrO}_{2}$, respectively; in agreement with experiments (see references in Table 1). Using the experimental band gap values in Table 1 allows one to obtain the $\alpha$ value leading to calculated values consistent with experiments, which we recall as optimal. Not surprisingly the optimal values for each one of the oxides studied in the present work is different (Table 1).

In order to properly discuss the results in Table 1 , it is necessary to caution that comparing theoretical band gaps with experimental data is 
not free of controversy. ${ }^{72-75}$ As indicated in the previous section, the presently calculated band gaps are computed as the energy difference between the valence band maximum (VBM) and the conduction band minimum (CBM) obtained by self-consistently solving the one electronic Kohn-Sham equations and thus neglecting many-body effects in the quasi particle excitations. On the other hand, all selected experimental band gaps were measured by different techniques: Ultraviolet-visible (UV) spectroscopy in the cases of $\mathrm{Cu}_{2} \mathrm{O}, \mathrm{TiO}_{2}, \mathrm{SnO}_{2}$, and $\mathrm{ZnO}_{2}$, electron-energy-loss spectroscopy (EELS) for $\mathrm{SrTiO}_{2}$ and $\mathrm{ZrO}_{2}$, and reflectance spectroscopy for $\mathrm{MgO}$. In these experiments, the electron and hole pair, called exciton, could be generated from incident light or an electron collision; there are several kinds of EELS (transmission, reflection, aloof and highresolution EELSs, and so on). ${ }^{76-78}$ Herein we only discussed the cases for the energy loss occurring from the surface inter-band transitions. Thus, additional contributions for electron-hole pair electrostatic interaction have to be taken into account, when one wishes a more accurate comparison between the experimental and theoretical band gaps. ${ }^{73}$ HSE calculations based on generalized Kohn-Sham scheme would give the calculated band gap excluding electron-hole pair binding energy. Thus, HSE band gap might be more near to the fundamental band gap. However, as a practical matter, HSE band gap also should be understood as a reasonable estimate of the experimental optical gap. This is justified because the optical and fundamental gaps in semiconductors are very close, as argued by Scuseria et al. $^{79}$ In this sense, it is understandable that the calculated results below show that the present theoretical estimates of band gaps are accurate enough.

\section{Relationship between the optimal HF mixing parameter and experimental dielectric constant}

From results in Table 1 it is clear that, with $w$ parameter $0 \AA^{-1}$, the optimal $\alpha$ values range from 0.119 to 0.293 , indicating that a universal hybrid functional able to reproduce the experimental band gaps is not achievable. In order to explain the need of using different values of $\alpha$ to reproduce the band gap of different materials, Fiorentini and Baldereschi, ${ }^{80}$ suggested that $\alpha$ is related to the inverse of the static dielectric constant $(1 / \varepsilon \infty)$ of the material. ${ }^{81}$ Experimental dielectric constant

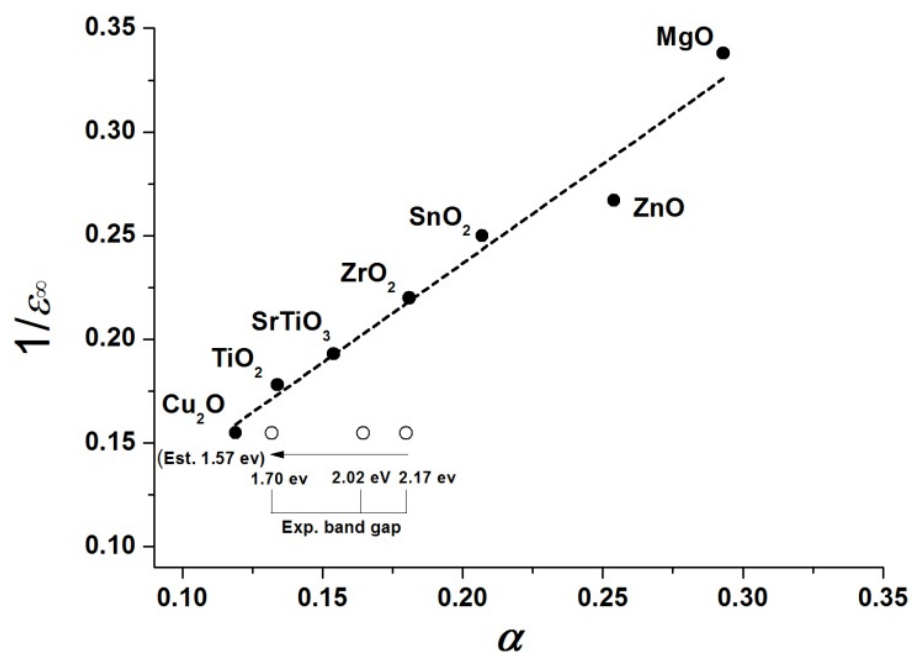

Figure 1. Correlation between the optimal mixing parameter $\alpha$ and the inverse of the experimental dielectric constants $(1 / \varepsilon \infty)$ for metal oxides under scrutiny. 
values and of its inverse $\left(1 / \varepsilon^{\infty}\right)$ are reported in Table 1. Herein, it is noted that the average values of static dielectric constants were used, when there are several experimental data reported for optically anisotropic oxides. The relationship between calculated optimal $\alpha$ and experimental $1 / \varepsilon^{\infty}$ is plotted in Figure 1 . Indeed, the relationship is almost linear and a proper fit leads to $\alpha=0.958 \times(1 / \varepsilon \infty)+0.045$ with $R^{2}=0.965$. This finding supports the present estimate of the band gaps and reinforces the interpretation of Marques et al., ${ }^{81}$ although it leaves the role of the $w$ parameter open.

The case of $\mathrm{Cu}_{2} \mathrm{O}$ deserves, however, a separate discussion since when we plotted the graph of Figure 1, we realized that previously reported band gaps measured by optical transition considerably deviated from the trend in Figure 1. Interestingly, the reported experimental band gap has been decreasing from the earlier measurements: $2.17 \mathrm{eV}$ in 1966, ${ }^{53} 2.02 \mathrm{eV}$ in $1970,{ }^{52}$ and $1.70 \mathrm{eV}$ in $1994 .^{54}$ The different values are marked as open circles in Figure 1. The differences in the reported experimental values of the band gap of $\mathrm{Cu}_{2} \mathrm{O}$ might be due to difficulties in growing stoichiometric and clean $\mathrm{Cu}_{2} \mathrm{O}$ samples, ${ }^{82}$ and to fact that the band gap of this material has the character of optically forbidden-direct transition because of the state symmetries of VBM and CBM having $\mathrm{Cu} 3 d$ - and $\mathrm{Cu} 4 s$-like characters, respectively. ${ }^{83}$ The most recent and carefully done UV experiments for a $\mathrm{Cu}_{2} \mathrm{O}$ thin film supported on $\mathrm{MgO}(100)$ reported a value of $1.70 \mathrm{eV}$. However, it is expected that this optical band gap would be wider than the ideal band gap of $\mathrm{Cu}_{2} \mathrm{O}$, because optical transitions between the band-edge states are also forbidden. Thus, we decided to get an estimate from this minimum value of $1.70 \mathrm{eV}$ by considering the reported correction energy, calculated as the difference between the forbidden transition energy and the first allowed transition energy, sized to be around $0.13 \mathrm{eV}^{83}$ Interestingly, our estimated value of minimum band gap $1.57 \mathrm{eV}$ (1.70 eV minus 0.13
eV) lines up the optimal $\alpha$ value, showing the remarkable nice fit to the plot in Figure 1.

\section{Optimal $\alpha: w$ trends of HSE hybrid functional reproducing experimental band gap value}

The above discussion highlighted a clear relationship between the band gap and $\alpha$ parameter, excluding the screening effect. Moreover, by comparing the optimal $\alpha$ parameters with the inverse experimental dielectric constant values, indirectly one can state that present selection of experimental band gap values seems to be reasonable. This setting might become an important first step to precisely explore the space for $\alpha$ and $w$ parameters of HSE functional. Here, one must point out that range separated functionals such as HSE introduce a physically meaningful additional parameter $(w)$ controlling the range where Fock exchange needs to switched off. In order to investigate more in detail the combined effect of $\alpha$ and $w$ and to find a possible combination working for all the materials studied in the present work we carried out a series of calculations to find the optimal $\alpha$ for different values of $w$, results summarized in Figure 2 and the complete set of data points included in Table S2 of Supporting Information.

The curves in Figure 2 correspond to $\alpha: w$ pairs which, for each material, exactly reproduce the experimental ban gap, as collected in Table 1. Otherwise, on the basis of each line, the calculated band gap values on the up-left are underestimated, and overestimated on the bottom-right. On two dimensional space composed of $\alpha$ and $w$ axes, the point for $\alpha=$ 0.25 and $w=0 \AA^{-1}$ formally is corresponding to the PBEO functional. Interestingly, PBEO correctly describes the band gap of $\mathrm{ZnO}$ as reported in previous studies, ${ }^{84,42}$ yet underestimates the band gap of $\mathrm{TiO}_{2}, \mathrm{SrTiO}_{3}$, $\mathrm{Cu}_{2} \mathrm{O}, \mathrm{SnO}_{2}$, and $\mathrm{ZrO}_{2}$, while overestimates that of $\mathrm{MgO}$. Let us now focus on the values with $\alpha=$ 0.25 and $w=0.21 \AA^{-1}$, which correspond to the so-called HSEO6 functional. This functional properly describes the band gap of $\mathrm{ZrO}_{2}$ as 


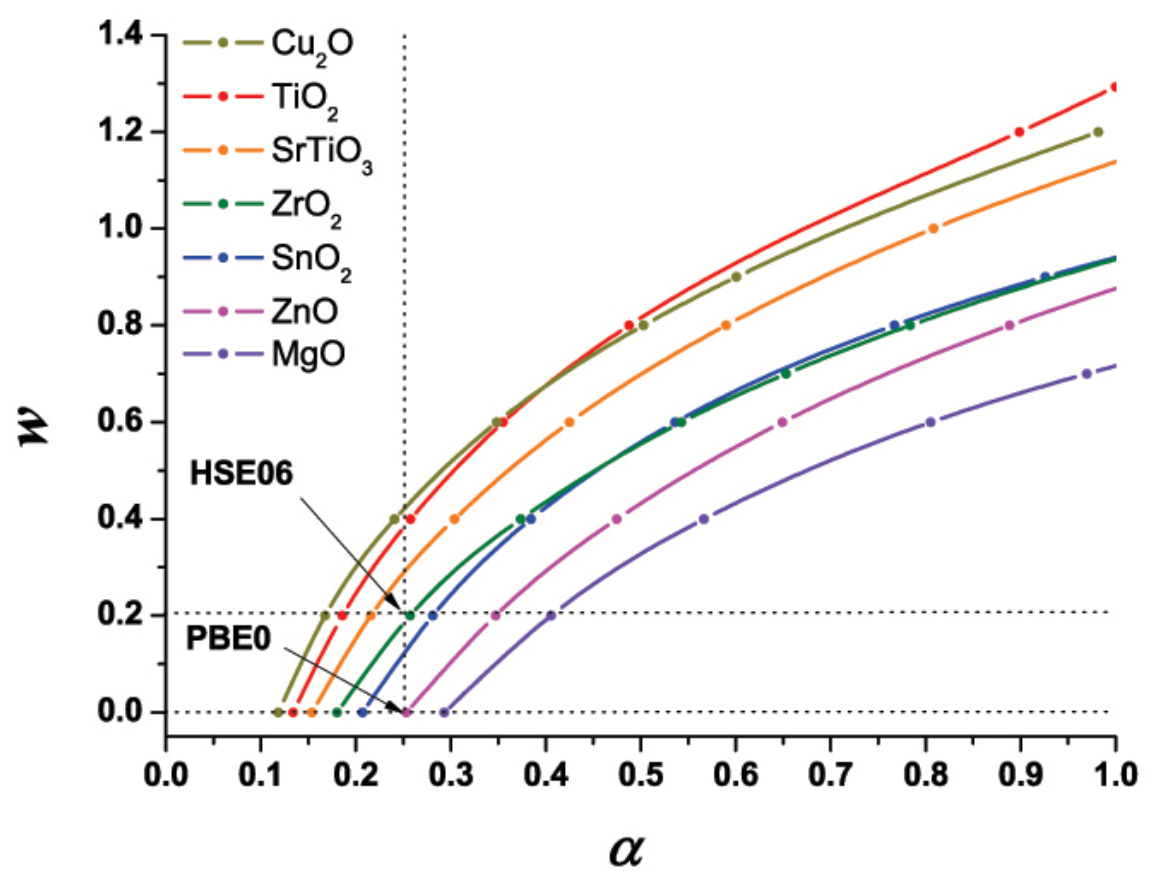

Figure 2. Optimal $\alpha: w$ trend of HSE hybrid functional reproducing the experimental band gap for 7 metal oxides.

described in previous studies, ${ }^{85,42}$ but underestimates the band gap of $\mathrm{TiO}_{2}, \mathrm{SrTiO}_{3}$, $\mathrm{Cu}_{2} \mathrm{O}$ oxides, while overestimating that of $\mathrm{SnO}_{2}$, $\mathrm{ZnO}$, and $\mathrm{MgO}$. From Figure 2 it appears that the success of HSEO6 in describing band gaps, either fundamental of optical, for various semiconductors, ${ }^{35}$ precisely is due to the fact that it lies on the central part of the curve rainbow.

On the other hand, for a given $\alpha$ value, such as 0.25 chosen for PBE0, it appears that different amounts of screening parameters are required to reproduce band gaps for the different metal oxides: around $0.4 \AA^{-1}$ for $\mathrm{Cu}_{2} \mathrm{O}$ and $\mathrm{TiO}_{2}$, around $0.3 \AA^{-1}$ for $\mathrm{SrTiO}_{3}, 0.2 \AA^{-1}$ for $\mathrm{ZrO}_{2}$, and around $0.1 \AA^{-1}$ for $\mathrm{SnO}_{2}$, respectively. This is not surprising since the screening needs to be also related to the electric response of the material. Hence, one could argue that it is more appropriate to stay with a particular amount of Fock exchange but tuning $w$ instead. However, it is noticeable that for $\mathrm{MgO}$ using any values of screening parameter $(w)$ with a given $\alpha$ value as 0.25 would not solve the band gap problem of underestimation. This is in agreement with the failure of HSE functional using $\alpha=0.25$ for large-gap insulators. ${ }^{81}$ In fact, Figure 2 shows that $\alpha>0.3$ is necessary to reproduce the band gap of $\mathrm{MgO}$ within HSE formalism. This is also in line with the previously suggested HSE12 and HSE12s functionals, having $\alpha=0.313$ and $\alpha=$ 0.425 , respectively, with different $w$ parameters to minimize overall error for diverse physical properties of broad range of solids, including MgO. ${ }^{31}$

As stated, from Figure 2 it is clear that there is an infinite number of $\alpha: w$ pairs reproducing the experimental band gap of a given oxide, but it is worth to highlight that the overestimation by applying a large $\alpha$ value is counteracted by increasing the $w$ screening. Following this rule, each metal oxide tends to exhibit a particular curvature of the $\alpha: w$ plot compatible with the experimental band gap value. Figure 2 also shows that, unfortunately, there is no single $\alpha: w$ pair describing all materials, although this seems possible for pairs of metal oxides. Indeed, cross points are found at the combination of $(\alpha$ $\left.=0.4: w=0.68 \AA^{-1}\right)$ and $\left(\alpha=0.47: w=0.52 \AA^{-1}\right)$ for $\mathrm{Cu}_{2} \mathrm{O}-\mathrm{TiO}_{2}$ and $\mathrm{ZrO}_{2}-\mathrm{SnO}_{2}$, respectively. In 

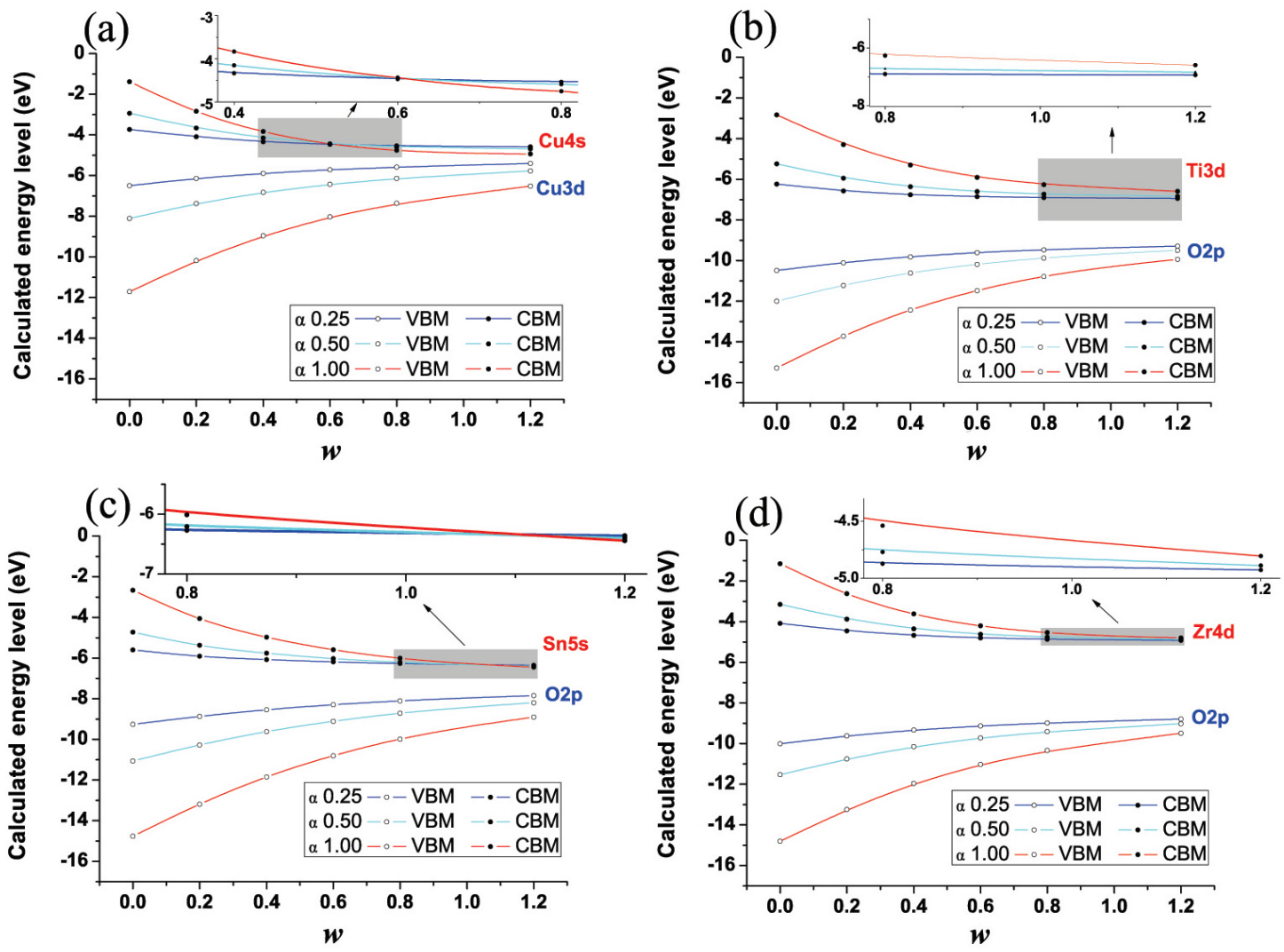

Figure 3. VBM and $\mathrm{CBM}$ (in eV) for $\mathrm{Cu}_{2} \mathrm{O}$ (a), $\mathrm{TiO}_{2}$ (b), $\mathrm{SnO}_{2}$ (c), and $\mathrm{ZrO}_{2}$ (d), as a function of $w$ for three values of $\alpha$ and screen parameter $w$.

this regard, these particular setups of the range separated HSE functional could be used to describe these binary metal oxide systems regarding the band gap. To shed light on the underlying reason for the existence of the crossing points for $\mathrm{Cu}_{2} \mathrm{O}-\mathrm{TiO}_{2}$ and $\mathrm{ZrO}_{2}-\mathrm{SnO}_{2}$ couples, we analyze the changes of energy levels for VBM and CBM of these oxides induced by $w$ for fixed values of $\alpha$. Figure 3 shows these trends for $\mathrm{Cu}_{2} \mathrm{O}, \mathrm{TiO}_{2}, \mathrm{ZrO}_{2}$, and $\mathrm{SnO}_{2}$.

Most notable feature of Figure 3 is that, for $\alpha$ values of $0.25,0.50$, and 1.00 , the effect of $w$ on the CBM lowering is different for the different metal oxides. Comparing $\mathrm{TiO}_{2}$ to $\mathrm{Cu}_{2} \mathrm{O}$, the $\mathrm{CBM}$ lines cross around $w=0.6 \AA^{-1}$ for the three $\alpha$ values. This cross point is also found for $\mathrm{SnO}_{2}$ at around $w=1.1 \AA^{-1}$ (see inset in Figure 3), whereas for $\mathrm{TiO}_{2}$ and $\mathrm{ZrO}_{2}$ the cross point occurs beyond $w=1.2 \AA^{-1}$. Interestingly, for
$\mathrm{Cu}_{2} \mathrm{O}$ and $\mathrm{SnO}_{2}$ the $\mathrm{CBM}$ is composed of $\mathrm{Cu} 4 \mathrm{~s}$ and $\mathrm{Sn} 5 \mathrm{~s}$ states, ${ }^{86,87}$ whereas for $\mathrm{TiO}_{2}$ and $\mathrm{ZrO}_{2}$ the CBM is dominated by $\mathrm{Ti} 5 d$ and $\mathrm{Zr} 4 d$ states. $^{34,88}$ This seems to indicate that, not surprisingly, the screening affects $s$ and $d$ bands differently. Figure S2 shows the CBM cross points for $\mathrm{ZnO}_{2}$ and $\mathrm{MgO}$ possessing both a CBM of $s$-like character. ${ }^{89,90}$ On the other hand, no cross point is found for $\mathrm{SrTiO}_{3}$ with a CBM of $3 d$ character. Consequently, the band energy levels are influenced by $\alpha$ and $w$ but also by the orbital character of the involved energy states. Thus, the difference of orbital character for energy state is likely to be behind the matching points of $\mathrm{Cu}_{2} \mathrm{O}-\mathrm{TiO}_{2}$ and $\mathrm{ZrO}_{2}-\mathrm{SnO}_{2}$ pairs. It should be mentioned that this is not likely to be the sole reason, or a general feature, since other crossing points should be observed and validated in future works. 
Finally, it is interesting to note that starting from pure Hartree-Fock ( $\alpha=1.0$ ) it would be possible to reproduce the band gap of the oxides using the appropriate screening factor, implying that $\mathrm{SR}$ is only described by $\mathrm{HF}$ exchange and LR only by PBE exchange while adding the appropriate correlation functional. From equation (4), the form can be derived as

$E_{x c}^{S X-P B E}=E_{x}^{H F, S R}(w)+E_{x}^{P B E, L R}(w)+E_{c}^{P B E}$

This is similar to the SX-LDA, proposed to yield improved band gaps by including screened exchange. ${ }^{7}$ Basically, exchange and correlation have an unambiguous definition when using wave functions. ${ }^{91}$ It retakes the meaning of exchange and correlation used in wave function based methods where Hartee-Fock is a well defined method and electron correlation has the meaning of instantaneous electron-electron interactions beyond the mean (self-consistent) field approximation. Generally, increasing the Fock exchange can reduce the spurious selfinteraction but then it misses electronic correlation effects leading to an overestimation of the band gap. Thus, the lack of correlation in pure Hartree-Fock exchange makes the gap too large and the physics needed to reduce the gap is simply to add the electron correlation contribution. This is more or less taken with the different functionals incorporating the proper screening length. This is a reason why it may be appealing to compensate the error of the band gap by the balance between the correlation and screened Hartree-Fock with appropriate screening parameters reflecting the cancellation of the long-range correlation and exchange energies. Figure 2 indicates that the proper screening parameters on those points have material dependence as like a proper $\mathrm{HF}$ mixing parameter with no screening effect. Note that the expected proper screening parameters at $\alpha$ $=1.0$ depicted in Figure 2 is highly related to Thomas-Fermi (TF) screening parameters (in $\AA^{-1}$ ) of SX-LDA functional comprehensively studied by Clark and co-workers. ${ }^{92-94}$ The only difference between HSE scheme and SX-LDA is the replacement of the LDA form for correlation and long-range exchange with the PBE form, as discussed by Chelikowsky et al. ${ }^{31}$ Within a simply screened Hartree-Fock exchange plus correlation logic, $\mathrm{TiO}_{2}$ appears to need the highest value of the screening parameter to properly predict the band gap whereas $\mathrm{MgO}$ will need the smallest one. However, the underlying reason of this tendency and the description for the defect systems still remains to be fully understood.

\section{Conclusions}

The effect of the internal parameters ( $\alpha$ and $w$ ) of the range separated HSE hybrid functional incorporating scalar relativistic effects in the description of the electronic structure mainly through the optical band gap neglecting possible excitonic effects, has been studied in detail for a set of seven metal oxides including transition metals with incomplete $d$-shells transition metals ( $\mathrm{Ti}, \mathrm{Zr}$ ), with complete $d$-shells $(\mathrm{Cu}, \mathrm{Zn})$, and also considering non-transition metals ( $\mathrm{Mg}$ and $\mathrm{Sn}$ ). In this study, we mainly investigated the effect of two parameters on band gaps. This is because the band gap is the most sensitive property to different settings of HSE functional. In fact, calculations for anatase, $\mathrm{TiO}_{2}$ (see Tables S3 and S4 in Supporting Information) evidence that the effect of the two parameters on lattice parameters and formation enthalpy does not exceed $3 \%$. It is worth pointing out that our investigation of the effect of two parameters is in line with recent work of Garza and Scuseria precisely on the band gap of oxides. ${ }^{95}$

Neglecting the range separation, that is, for pure hybrid functionals, the calculated band gap is linearly correlated with the amount of Fock exchange included in the exchange part of the exchange-correlation potential, thus generalizing previous observations. ${ }^{74}$ This finding suggest that the screening parameter $w$ may not be needed, at least as far as the prediction of the band gap is concerned for metal oxides, although introducing this parameter facilitates convergence of the 
required calculations. In these cases, it appears that the amount of optimal Fock exchange corresponding to the selected experimental band gap linearly correlates with the inverse of the experimental dielectric constant of the material, again in agreement with previous works. ${ }^{80,81}$ From this step, we can confirm that our selection about the experimental band gap values, specifically optical gap values, are uniformly reasonable for the studied metal oxides.

The introduction of the screening parameter has an effect which is contrary to that of the amount of Fock exchange in such a way that increasing the value of the screening parameter reduces the calculated band gap value, this is one of the reasons why HSEO6 represent an improvement over PBEO for the calculation of band gaps, where a possible overestimation of the bandgap cannot be counteracted. However, compared to Fock exchange, the correlation between screening parameters and the calculated band gap tends to be changed from inverse proportional to exponential decay as the percentage of Fock exchange increases. Thus, only using the linear relationship between the Fock exchange and the calculated band gap would be a straightforward way to find the optimal set of two parameters via linear inter/extrapolation method.

At last, it is found that there is an infinite number of $\alpha: w$ combinations which, for a given metal oxide, reproduce its experimental band gap. This makes it difficult to define a unique range separated HSE-like functional able to properly describe the band gap of a given family of oxides, even though matching points for $\mathrm{Cu}_{2} \mathrm{O}-\mathrm{TiO}_{2}$ and $\mathrm{ZrO}_{2}-\mathrm{SnO}_{2}$ pairs are expected to exist. From the energy state analysis regarding these, it is shown that the subtle curvature difference on $\alpha: w$ curves between oxides might be related with the orbital characteristic of the involved energy states. Consequently, our results provide an evidence that the band energy levels for oxides are influenced by $\alpha$ and $w$ but also by the orbital character within HSE functional formalism. However, the details of the electric response for the electron density according to orbital character should be explored depending on the two parameters of HSE functional. Additionally, the present systematic study shows that it is possible to reproduce the band gap of oxides starting from the Hartree-Fock approximation, and adding correlation through the appropriate functional, provided the short range part of the Fock exchange is properly screened. This will put the difference in the material rather than on the method, which seems physically appealing. These conclusions are presented for metal oxides, it is sensible to think that conclusions would remain valid for other semiconductors and isolators, such as sulfides and selenides.

\section{Acknowledgments}

This research was supported by the Spanish MINECO/FEDER CTQ2015-64618-R grant, in part by Generalitat de Catalunya (grants 2014SGR97 and XRQTC), and also by European Union's Horizon 2020 research and innovation programme under grant agreement No 676580 (the NOMAD Center of Excellence project). J.Y.L. acknowledges the financial support by Ministry of Science, ICT and Future Planning, subjected project to the project EDISON (Educationresearch Integration through Simulation On the Net, Grant No. 2012M3C1A6035359); K.C.K. acknowledges the financial support by Basic Science Research Program through the National Research Foundation of Korea (NRF) funded by the Ministry of Education (NRF2014R1A6A3A03056449); F.V. thanks the MINECO for a postdoctoral Ramón y Cajal (RyC) research contract (RYC-2012-10129); O.L.G is grateful to the Universitat de Barcelona for a predoctoral grant, and F.I. acknowledges additional support from the 2015 ICREA Academia Award for Excellence in University Research. Computational time at the Marenostrum supercomputer has been provided by the Barcelona Supercomputing Centre through grants from Red Española de Supercomputación and the COMPHOTOCAT 
project 2014112608 of the Partnership for Advanced Computing in Europe (PRACE).

Keywords: Metal oxides, Band gap, DFT, Hartree-Fock exchange, Screen parameter, HSE functional

Additional Supporting Information may be found in the online version of this article.

\section{References and Notes}

1. G. Koster, Epitaxial growth of complex metal oxides; Elsevier: Boston, MA, 2015.

2. J. Bednorz, K. Müller, Z. Phys. B, 1986, 64, 189-193.

3. M. Imada, A. Fujimori, Y. Tokura, Rev. Mod. Phys., 1998, 70, 1039.

4. X. Yu, T. J. Marks, A. Facchetti, Nat. Mater., 2016, 15, 383-369.

5. T. Grewe, M. Meggouh, H. Tüysüz, Chem.Asian J., 2016, 11, 22-42.

6. L. Yuan, C. Han, M.-Q. Yang, Y.-J. Xu, Int. Rev. Phys. Chem., 2016, 35, 1-36.

7. D. M. Bylander, L. Kleinman, Phys. Rev. B, 1990, 41, 7868.

8. V. N. Staroverov, G. E. Scuseria, J. Tao, J. P. Perdew, Phys. Rev. B, 2004, 69, 075102.

9. C. Sousa, S. Tosoni, F. Illas, Chem. Rev., 2013, 113, 4456-4495.

10. J. P. Perdew, A. Zunger, Phys. Rev. B, 1981, 23, 5048.

11. A. J. Cohen, P. Mori-Sanchez, W. Yang, Chem. Rev., 2012, 112, 289-320.

12. A. D. Becke, J. Chem. Phys., 1993, 98, 56485652.

13. P. Perdew, M. Ernzerhof, K. Burke, J. Chem. Phys., 1996, 105, 9982-9985.

14. C. Adamo, V. Barone, J. Chem. Phys., 1999, $110,6158-6170$.

15. S. V. Levchenko, X. Ren, J. Wieferink, R. Johanni, P. Rinke, V. Blum, M. Scheffler, Comput. Phys. Commun., 2015, 192, 60-69.

16. J. Paier, R. Hirschl, M. Marsman, G. Kresse, J. Chem. Phys., 2005, 122, 234102.

17. T. Bredow, A. R. Gerson, Phys. Rev. B, 2000, 61, 5194.

18. J. Muscat, A. Wander, N. M. Harrison, Chem. Phys. Lett., 2001, 342, 397.
19. K. N. Kudin, G. E. Scuseria, R. L. Martin, Phys. Rev. Lett., 2002, 89, 266402.

20. I. de P. R. Moreira, F. Illas, R. L. Martin, Phys. Rev. B, 2002, 65, 155102.

21. C. Franchini, V. Bayer, R. Podloucky, J. Paier, G. Kresse, Phys. Rev. B, 2005, 72, 045132.

22. J. L. F. Da Silva, M. V. Ganduglia-Pirovano, J. Sauer, V. Bayer, G. Kresse, Phys. Rev. B, 2007, 75, 045121.

23. J. Heyd, G. E. Scuseria, M. Ernzerhof, J. Chem. Phys., 2003, 118, 8207-8215.

24. J. Heyd, G. E. Scuseria, M. Ernzerhof, J. Chem. Phys., 2006, 124, 219906(E).

25. J. Heyd, G. E. Scuseria, J. Chem. Phys., 2004, 120, 7274-7280.

26. J. Heyd, G. E. Scuseria, J. Chem. Phys., 2004, $121,1187-1192$.

27. A. V. Krukau, O. A. Vydrov, A. F. Izmaylov, G. E. Scuseria, J. Chem. Phys., 2006, 125, 224106-224106.

28. J. Heyd, J. E. Peralta, G. E. Scuseria, R. L. Martin, J. Chem. Phys., 2005, 123, 174101.

29. J. E. Peralta, J. Heyd, G. E. Scuseria, R. L. Martin, Phys. Rev. B, 2006, 74, 073101.

30. I. D. Prodan, G. E. Scuseria, R. L. Martin, Phys. Rev. B, 2006, 73, 045104.

31. J. E. Moussa, P. A. Schultz, J. R. Chelikowsky, J. Chem. Phys., 2012, 136, 204117.

32. J. P. Perdew, K. Burke, M. Ernzerhof, Phys. Rev. Lett., 1996, 77, 3865.

33. M. Ernzerhof, G. E. Scuseria, J. Chem. Phys., 1999, 110, 5029-5036.

34. K. C. Ko, O. Lamiel-García, J. Y. Lee, F. Illas, Phys. Chem. Chem. Phys., 2016, 18, 1235712367.

35. B. G. Janesko, T. M. Henderson, G. E. Scuseria, Phys. Chem. Chem. Phys., 2009, 11, 443-454.

36. E. N. Brothers, A. F. Izmaylov, J. O. Normand, V. Barone, G. E. Scuseria, J. Chem. Phys., 2008, 129, 011102.

37. Y. F. Zhang, W. Lin, Y. Li, K. N. Ding, J. Q. Li, J. Phys. Chem. B, 2005, 109, 19270-19277.

38. M.-A. Haa, A. N. Alexandrova, J. Chem. Theory Comput., 2016, 12, 2889-2895.

39. A. Janotti, J. Varley, P. Rinke, N. Umezawa, G. Kresse, C. Van de Walle, Phys. Rev. B, 2010, 81, 085212. 
40. D. Koller, P. Blaha, F. Tran, J. Phys.: Condens. Matter., 2013, 25, 435503.

41. J. H. Skone, M. Govoni, G. Galli, Phys. Rev. B, 2016, 93, 235106.

42. M. Gerosa, C. E. Bottani, L. Caramella, G. Onida, C. Di Valentin, G. Pacchioni, Phys. Rev. B, 2015, 91, 155201.

43. S. Lany, Phys. Rev. B, 2013, 87, 085112.

44. V. Stevanovic, S. Lany, D. S. Ginley, W. Tumas, A. Zunger, Phys. Chem. Chem. Phys., 2014, 16, 3706-3714.

45. www.icsd.ill.fr/icsd/index.php

46. www.materialsproject.org

47. V. Blum, R. Gehrke, F. Hanke, P. Havu, V. Havu, X. Ren, K. Reuter, M. Scheffler, Comput. Phys. Commun., 2009, 180, 21752196.

48. V. Havu, V. Blum, P. Havu, M. Scheffler, J. Comput. Phys., 2009, 228, 8367-8379.

49. I. Y. Zhang, X. G. Ren, P. Rinke, V. Blum, M. Scheffler, New J. Phys., 2013, 15, 123033.

50. E. van Lenthe, E. J. Baerends, J. G. Snijders, J. Chem. Phys., 1994, 101, 9783-9792.

51. S. B. Ogale, P. G. Bilurkar, N. Mate, S. M. Kanetkar, N. Parikh, B. Patnaik, J. Appl. Phys., 1992, 72, 3765-3769.

52. S. P. Tandon, J. P. Gupta, Phys. Status. Solidi. $B, 1970,37,43-45$.

53. S. Brahms, S. Nikitine, J. P. Dahl, Phys. Lett., 1966, 22, 31-33.

54. I. Grozdanov, Mater. Lett., 1994, 19, $281-$ 285.

55. M. O’Keeffe, J. Chem. Phys., 1963, 39, 17891793.

56. D. O. Scanlon, C. W. Dunnill, J. Buckeridge, S. A. Shevlin, A. J. Logsdail, S. M. Woodley, C. R. A. Catlow, M. J. Powell, R. G. Palgrave, I. P. Parkin, Nat. Mater., 2013, 12, 798-801.

57. H. Tang, F. Lévy, H. Berger and P. E. Schmid, Phys. Rev. B, 1995, 52, 7771.

58. S. Wemple, J. Chem. Phys., 1977, 67, 21512168.

59. A. Benrekia, N. Benkhettou, A. Nassour, M. Driz, M. Sahnoun, S. Lebègue, Physica $B$, 2012, 407, 2632-2636.

60. W. Zhong, R. D. King-Smith, D. Vanderbilt, Phys. Rev. Lett., 1994, 72, 3618.
61. L. K. Dash, N. Vast, P. Baranek, M.-C. Cheynet, L. Reining, Phys. Rev. B, 2004, 70, 245116.

62. H. French, S. J. Glass, F. S. Ohuchi, Y. N. Xu and W. Y. Ching, Phys. Rev. B, 1994, 49, 5133.

63. Simply calculated from well-known refractive index value 2.13, using the relation $\varepsilon_{0}=\mathrm{n}^{2}$.

64. O. Madelung, Semiconductors-basic data; Springer Science \& Business Media: Berlin, 2012.

65. M. Dou, C. Persson, J. Appl. Phys., 2013, 113, 083703.

66. K. H. Hellwege; O. Madelung, Numerical Data and Functional Relationships in Science and Technology, Landolt-Börnstein, New Series, Group III, Vol. 17, Part a, Springer: Berlin, 1982.

67. W. Y. Liang, A. D. Yoffe, Phys. Rev. Lett., 1968, 20, 59.

68. D. C. Reynolds, D. C. Look, B. Jogai, C. W. Litton, G. Cantwell, W. C. Harsch, Phys. Rev. $B, 1999,60,2340$.

69. N. Ashkenov, B. N. Mbenkum, C. Bundesmann, V. Riede, M. Lorenz, D. Spemann, E. M. Kaidashev, A. Kasic, M. Schubert, M. Grundmann, G. Wagner, H. Neumann, V. Darakchieva, H. Arwin, B. Monemar, J. Appl. Phys., 2003, 93, 126-133.

70. R. C. Whited, C. J. Flaten, W. C. Walker, Solid State Commun., 1973, 13, 1903-1905.

71. D. R. Lide, CRC handbook of chemistry and physics, CRC press: New York, 2004.

72. H. Jiang, R. I. Gomez-Abal, P. Rinke, M. Scheffler, Phys. Rev. B, 2010, 81, 085119.

73. J.-L. Bredas, Materials Horizons, 2014, 1, 1719.

74. D. Cho, K. C. Ko, O. Lamiel-García, S. T. Bromley, J. Y. Lee, F. Illas, J. Chem. Theory Comput., 2016, 12, 3751-3763.

75. L. Kronik, T. Stein, S. Refaely-Abramson, R. Baer, J. Chem. Theory Comput., 2012, 8, 1515-1531.

76. R. F. Egerton, Rep. Prog. Phys., 2009, 72, 016502.

77. B. Reed, M. Sarikaya, Ultramicroscopy, 2002, 93, 25-37. 
78. G. Bracco; B. Holst, Surface science techniques, Springer Science \& Business Media: Berlin, 2013.

79. T. M. Henderson, J. Paier, G. E. Scuseria, Phys. Status. Solidi. B, 2011, 248, 767-774.

80. V. Fiorentini, A. Baldereschi, Phys. Rev. B, 1995, 51, 17196.

81. M. A. L. Marques, J. Vidal, M. J. T. Oliveira, L. Reining, S. Botti, Phys. Rev. B, 2011, 83, 035119.

82. D. Tahir, S. Tougaard, J. Phys.: Condens. Matter, 2012, 24, 175002.

83. D. C. Reynolds, Excitons: their properties and uses, Elsevier: New York, 2012.

84. D. Gryaznov, E. Blokhin, A. Sorokine, E. A. Kotomin, R. A. Evarestov, A. BussmannHolder, J. Maier, J. Phys. Chem. C, 2013, 117, 13776-13784.

85. A. Sinhamahapatra, J.-P. Jeon, J. Kang, B. Han, J.-S. Yu, Sci. Rep., 2016, 6, 27218.

86. B. K. Meyer, A. Polity, D. Reppin, M. Becker, P. Hering, P. J. Klar, T. Sander, C. Reindl, J. Benz, M. Eickhoff, C. Heiliger, M. Heinemann, J. Bläsing, A. Krost, S.
Shokovets, C. Müller, C. Ronning, Phys. Status. Solidi. B, 2012, 249, 1487-1509.

87. M. Batzill, U. Diebold, Prog. Surf. Sci., 2005, 79, 47-154.

88. C. Ricca, A. Ringuedé, M. Cassir, C. Adamo, F. Labat, J. Comput. Chem., 2015, 36, 9-21.

89. A. S. Alshammari, L. Chi, X. Chen, A. Bagabas, D. Kramer, A. Alromaeh, Z. Jiang, RSC Adv., 2015, 5, 27690-27698.

90. R. A. Èvarestov, Theoretical modeling of inorganic nanostructures: Symmetry and ab-initio calculations of nanolayers, nanotubes and nanowires, Springer: Berlin, 2015.

91. G. C. Lie, E. Clementi, J. Chem. Phys., 1974, 60, 1275-1287.

92. S. J. Clark, J. Robertson, Phys. Rev. B, 2010, $82,085208$.

93. S. J. Clark, J. Robertson, Phys. Status. Solidi. $B, 2011,248,537-546$.

94. G. Yuzheng, R. John, J. C. Stewart, J. Phys.: Condens. Matter, 2015, 27, 025501.

95. A. J. Garza, G. E. Scuseria, J. Phys. Chem. Lett. 2016, 7, 4165-4170. 


\section{GRAPHICAL ABSTRACT}

Francesc Viñes, Oriol Lamiel-García, Kyoung Chul Ko, * Jin Yong Lee and Francesc Illas*

Systematic study of the effect of HSE functional internal parameters on the electronic structure and band gap of a representative set metal oxides

The effect of the amount of Fock exchange and of the screening parameter defining the range separated HSE type hybrid functional is systematically studied for a series of seven metal oxides to define the combination of two parameters reproducing the band gap.

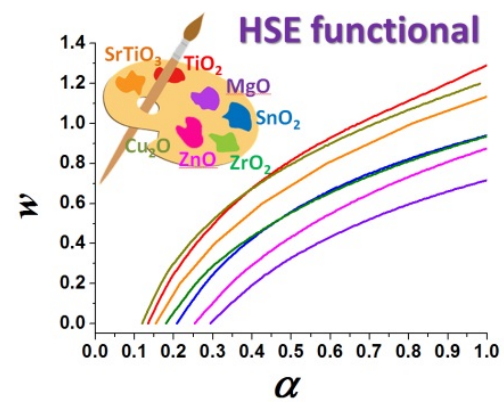

